\title{
PARASPHENOID UND BASITEMPORALIA DER SÄUGETIERE
}

\author{
VON \\ P. N. VAN KAMPEN, \\ LEIDEN.
}

(Mit 1 Figur im Tekst)

\begin{abstract}
Seitdem ich unter Leitung von Prof. MAx WeBer meine Doctorarbeit über die Tympanalgegend des Säugetierschädels geschrieben habe, hat dieses Thema mich immer gefesselt und ich freue mich meinem verehrten Lehrer jetzt eine kleine Arbeit widmen zu können, welche sich mit einem benachbarten Abschnitt des Säugetierschädels beschäftigt.

Unter den „neuen Deutungen auf dem Gebiete der Lehre vom Säugetierschädel”, welche GAupp auf Grund seiner schönen und vielumfassenden Untersuchungen gegeben hat, giebt es eine, welche keine allgemeine Einstimmung gefunden, und womit er auch nach meiner Meinung, welche ich im Folgenden auseinanderzusetzen beabsichtige, nicht das Richtige getroffen hat. Es ist diejenige, nach welcher die bis daher immer als „Pterygoide" gedeuteten Knochen der Ditremen Säugetiere nicht von den Pterygoiden, sondern von den Seitenteilen des Parasphenoid (Gaupp's „Parabasale”) der niederen Vertebraten herstammen soliten ${ }^{1}$ ).

In mehreren Arbeiten hat GAUPP diese-Ansicht verteidigt ${ }^{2}$ ) und Einige sind ihm darin gefolgt, indem sie sogar schon nicht mehr vom „Pterygoid”, sondern vom „Parasphenoid” der Säuger reden. Unter den Gegnern von GaUPP's Auffassung sind namentlich FUCHS ${ }^{3}$ ) und WaTson ${ }^{4}$ ) zu nennen.

Die Auffassung von GAUPP stützt sich auf seine Entdeckung eines Paares von kleinen Deckknochen, welche bei Beuteljungen von Echidna dorsal von den Palatina und medial von den Wurzeln der Alae temporales der Schädelbasis anliegen. Beim erwachsenen Tiere sind sie mit dem Sphenoid verwachsen und bilden einen Teil der sogenannten Proc. pterygoidei desselben. In ihrer Lage ähneln sie einerseits den Querschenkeln des Parasphenoid der Saurier, andrerseits den Pterygoiden der viviparen Säugetiere. Diese letzteren könnten demnach durch Vermittlung der kleinen Deckknochen von Echidna vom Parasphenoid hergeleitet werden. - Nachher hat WATSON in seiner oben zitierten Arbeit dieselben Knochen bei Ornithorhynchus aufgefunden.

Auch wenn man mit der von GaUPP vertretenen Auffassung der Homologie der Säugerpterygoide einstimmt, bleibt es aber doch fraglich, ob der Namen „Parasphenoid” für diese Knochen richtig gewählt ist. GAUPP hat gezeigt, dass die Querschenkel des Parasphenoid bei Lacerta selbständig verknöchern und weist darauf hin, dass solche selbständig entstehende laterale Teile des Parasphenoid auch bei anderen Sauropsiden beschrieben worden sind, und zwar bei Krokodilen von PARKER ${ }^{5}$ ), bei
\end{abstract}

1) Anat. Anz., Bd. XXVII, 1905.

2) Namentlich in seiner Bearbeitung des Echidna-Schädels in Semon's Forschungsreisen, Bd. III, Jena, 1908, und in den Anatomischen Heften, 1. Abt., Bd. XLII, 1911.

3) Verhand1. Anat. Ges., 1909; Arch. f. Anat. und Entw.-Gesch., 1909, Suppl.-Bd., und Anat. Anz., Bd. XXXVI, 1910.

4) Phil. Trans. R. Soc. London, B, vol. 207, 1916.

5) Transact. Zoolog. Soc. London, vol. XI, 1883. SHIINo (Anat. Hefte, I, Abt., Bd. L, 1914) hat diese Angabe bestätigt. 
Sphenodon von Schauinsland ${ }^{1}$ ), bei Vögeln von PARKer ${ }^{2}$ ) und SuSChKIN ${ }^{3}$ ). Gaupp sieht hierin eine Zerteilung („Primordialdivision”) des anfänglich einheitlichen Parasphenoid; mindestens ebenso wahrscheinlich erscheint jedoch die entgegengesetzte Auffassung, dass nämlich die selbständig ossifizierenden Seitenteile, PARKER's „Basitemporalia”, vom Ursprung an selbständige Skelettstücke gewesen sind. Ob deren Vorläufer schon bei Fischen und Amphibien aufzufinden sind, muss dahingestellt bleiben; vielleicht kommt' ein Paar der Pterygoidea der Fische als solche in Betracht. Wie dem auch sei, um Verwechslung zu entgehen wird es besser sein, für GAupP's "Seitenteile des Parasphenoid" den Namen „Basitemporalia” zu benützen; der phylogenetische Ursprung derselben kann dabei für uns ausser Betracht bleiben ${ }^{4}$ ).

Ein richtiges, ungepaartes Parasphenoid in rudimentärer Ausbildung ist bei Säugetieren aufgefunden worden von PARKER ${ }^{5}$ ) (bei Galeopithecus) und Fuchs ${ }^{6}$ ) (bei Didelphys). ToePLITZ ${ }^{7}$ ) hat es bei Didelphys nicht wiederfinden können; ich kann das Vorkommen aber sowohl bei Didelphys wie bei Galeopithecus bestätigen ${ }^{8}$ ).

Nach GAUPP soliten demnach unter den Säugetieren nur den Monotremen (Reptilien)pterygoide zukommen. Er denkt an die Möglichkeit die Entotympanica von höheren Säugetieren aus ihnen herzuleiten, aber es ist dies, wie ich schon früher auseinandersetzte ${ }^{9}$ ), wegen Unterschiede in der Lage nicht wahrscheinlich.

Es sind aber bei einigen Säugetieren andere Knochen beschrieben worden, welche für diese Frage in Betracht gezogen werden müssen. Sie liegen cerebral von den Palatina und (Säuger)pterygoiden und daher eben an derjenigen Stelle, wo Basitemporalia erwartet werden könnten.

An erster Stelle denke ich hierbei an ein Paar Knochen, welche PARKER ${ }^{10}$ ) bei Galeopithecus beschreibt und abbildet und nach ihm lateral von dem oben erwähnten medianen Parasphenoid zwischen Pterygoid und Schädelbasis liegen. "The pterygoid is very small, as in the Marsupials," schreibt er, "and this comes from the fact that what is generally, in the higher mammals, the upper or basicranial flange, is here, as in the Marsupials, a long, tongue-shaped mesopterygoid. This flat bone is sharp in front, where it wedges in between the palatine and pterygoid, and blunt behind, where it partly hides the basisphenoid; its front part hides the side of the presphenoid; it is gently arcuate, the convex side being toward the mid-line."

In einer Fussnote (S. 260) schreibt er überdies: „I shall have to describe a pair of bones found in membrane, and added to the basisphenoid, when I come to other and higher kinds of Eutheria; these have long been known in Man as the „lingulae sphenoidales”. I strongly suspect that they are the "basitemporals" - symmetrical remnants of the parasphenoid - so well seen in Birds and the Crocodiles."

Sonderbar ist es, dass PARKER die ",Mesopterygoidea” von Galeopithecus und den Marsupialia nicht mit den Basitemporalia in Verbindung zu bringen scheint, trotz der offenbaren Uebereinstimmung dieser Knochen mit denjenigen, welche er für die höheren Eutheria erwähnt und als Basitemporalia deutet. Vielleicht hält er das Vorkommen von Basitemporalia unvereinbar mit dem medianen Para-

1) Arch. f. mikr. Anat., Bd. LVI, 1900. Diese Angabe ist aber von FUchs (Anat. Anz., Bd. XXXVI, 1910) widersprochen.

2) Phil. Transact. R. Soc. London 1866 und (1869), 1870.

3) Nouv. Mém. Soc. Imp. Naturalistes de Moscou, vol. XVI, 1899.

4) Auf die abweichenden Betrachtungen von KeSTEVEn (Jrn. of Anat., vol. LIII, 1919), der die Reptilienpterygoide aus dem Parasphenoid der Amphibien entstehen lässt (die Pterygoidea der Amphibien sollten dann zu den Transversa geworden sein) braucht nicht näher eingegangen zu werden, weil sie keine Rechnung tragen mit den Basitemporalia, weder auch mit dem bei Schildkröten und Krokodilen nachgewiesenen Parasphenoid (sieh auch WATSON, Jrn. of Anat., vol. LIII, 1919).

5) Phil. Transact. R. Soc. London, vol. 176, 1885.

6) Anat. Anz., Bd. XXXII, 1908.

7) Zoologica, H. 70, 1920.

8) Wie FUCHS bemerkt, wird hierdurch die Hypothese von SUTTON, nach welcher das Parasphenoid der Säuger im Vomer gesucht werden müsste, hinfällig. Nur das stark ausgebildete, sich weit nach hinten erstreckende „Vomer" von Ornithorhynchus wäre vielleicht als Parasphenoid zu deuten.

9) Morph. Jahrb., Bd. XXXIV., 1905, S. 705. Sieh auch: VAN DER KLAAUUw, Ueber die Entwickelung des Entotympanicums, in: Tijdschr. Nederl. Dierk. Ver., (2), dl. XVIII, 1922.

10) 1. c. (1885), S. 253; Taf. 37, Fig. 1, 6, 8; Taf. 39, Fig. 1, 8. 
sphenoidrudiment von Galeopithecus. Aus seinen Beschreibungen und Abbildungen aber geht die Aehnlichkeit seiner Mesopterygoide mit den Basitemporalia deutlich hervor.

Die von PARKER beabsichtigte Beschreibung der Schädel von höheren Eutheria ist leider niemals erschienen. Was er meint mit "a pair of bones found in membrane, and added to the basisphenoid", geht vielleicht hervor aus einer Stelle in seinem "Morphology of the Skull” "). Er beschreibt dort bei Cavia eine lange, verknöcherte "lingula", welche sich von der Schädelbasis nach der Bulla tympanica erstreckt und an deren Spitze ein S-förmiger Knochen liegt, welcher später mit ihr verwachst und den „Basitemporalflügel” der Vögel repräsentiert. Dass auch die „Lingulae sphenoidales”. des Menschen hierher gehören sollen, wie PARKER andeutet, ist unwahrscheinlich, weil sie aus der knorpligen Schädelbasis hervorgehen.

Hingegen sind die sogenannten „Ossicula Bertini” (Conchae sphenoidales) des Menschen wahrscheinlich als Basitemporalia zu deuten, wenigstens insoweit sie als Belegknochen entstehen ${ }^{2}$ ).

Falls die Deutung der genannten Knöchelchen (welche demnach den Marsupialia, Galeopithecus, den Rodentia und dem Menschen zukommen und wohl noch allgemeiner verbreitet sein dürften) als Basitemporalia richtig ist, geht daraus hervor, dass GAUPP's Ansichten über die Säugerpterygoide nicht aufrecht erhalten werden können. Fuchs' Widerlegung wird dadurch bestätigt und die Säugerpterygoide sind den Reptilienpterygoiden homolog zu betrachten.

Auch bei einigen Insectivoren glaube ich das Vorkommen von Basitemporalia annehmen zu dürfen. Nur habe ich hier keine getrennte Anlage derselben finden können und die Beweisführung wird dadurch erschwert. Als Basitemporalia betrachte ich nämlich die Processus tympanici des Basisphenoid, welche vielen Insectivoren zukommen und in der Umgrenzung von Paukenhöhlen und Tubae Eustachii liegen (ebenso wie die Basitemporalia bei den Vögeln ${ }^{3}$ )). Ihre Entwicklung, teilweise schon von PARKER in seiner oben citierten Abhandlung über den Insectivoren-Schädel (1885) beschrieben, habe ich bei Erinaceus und Talpa genauer untersucht ${ }^{4}$ ). Sie entstehen hier erst spät: bei einem Embryo von Erinaceus $(12,5 \mathrm{~mm}$. Schnauze bis Scheitel, $37 \mathrm{~mm}$. Scheitel bis Schwanzwurzel), bei welchem die meisten Schädelknochen schon mehr oder weniger entwickelt sind, fehlen die Proc. tympanici noch vollständig. In einem ältern Stadium (Länge nicht angegeben) finde ich sie ebenfalls noch nicht deutlich entwickelt, aber doch wahrscheinlich vorhanden in der Gestalt von kleinen Knochenhöckern des Basisphenoid, welche mit den Pterygoiden verwachsen sind.

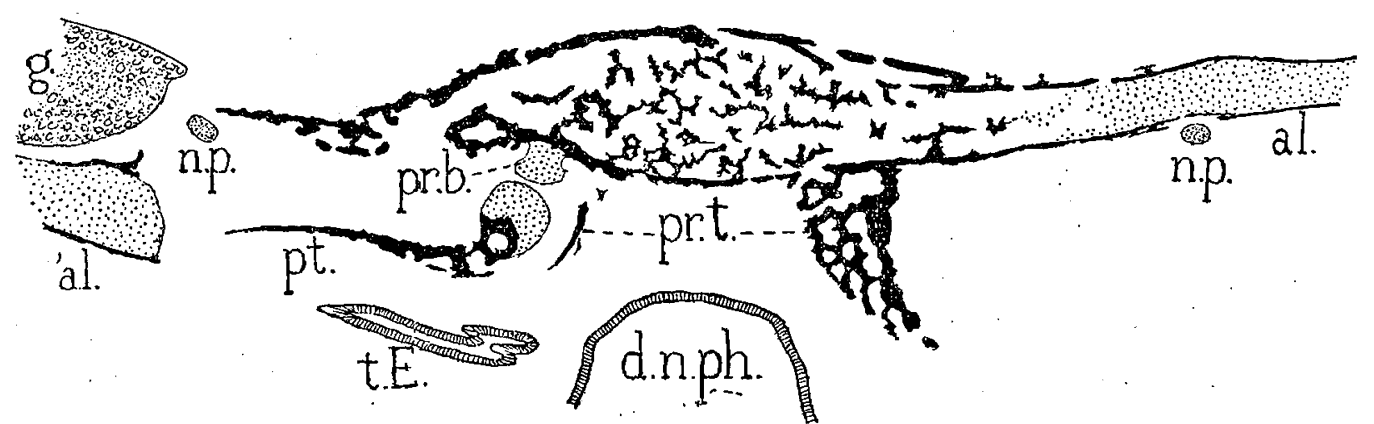

Fig. 1. Basisphenoid und Umgebung eines Embryo's. von Talpa europaea, Querschnitt (Knochen schwarz, Knorpel grobkörnig, Nerven feinkörnig, Epithel gestrichelt). Der Schnitt ist etwas schräg, und zwar hat er an der rechten Seite ein wenig mehr nach hinten getroffen als an der linken. - al. Ala temporalis; d. n. ph. Ductus nasopharyngeus; $g$. Ganglion Gasseri; n. p. Nervus parabasalis; pr. b. Proc. basipterygoideus (?); pr. t. Proc. tympanicus des Basisphenoid; pt. Pterygoid; t. E. Tuba Eustachii.

Auch bei einem gleichfalls schon weit entwickelten Embryo von Talpa (Schnauze bis Scheitel 23, Scheitel bis Schwanzwurzel $53 \mathrm{~mm}$.) ist der knöcherne Proc. tympanicus verschmolzen mit dem verknöcherten Basisphenoid (Fig. 1), nicht aber mit dem Pterygoid, welches hier frei bleibt; durch Knorpel ist das Basisphenoid getrennt vom Alisphenoid, welches als Knochenkern in der fast horizontal liegenden Ala temporalis ausgebildet ist.

1) Deutsche Uebersetzung (1879), S. 297.

2) Cf. Von SPEE, in: Bardeleben, Handbuch der Anatomie des Menschen, 1. Bd., 2 Abt., 1896, S. 146.

3) Suschkin, 1. c., S. 24.

4) Das hierzu benützte Material verdanke ich grösstenteils Herrn Dr. D. DE LANGE, Direktor des Embryologischen Institutes in Utrecht. 
Obgleich also eine getrennte Anlage der Proc. tympanici nicht wahrscheinlich ist, glaube ich sie dennoch wegen der übereinstimmenden Lage und des allostotischen Ursprunges als Basitemporalia. betrachten $z \mathfrak{u}$ dürfen, welche dann entweder vom Anfange an im Zusammenhang mit dem Basisphenoid verknöchern ') oder sehr bald nach ihrer Anlage mit demselben verwachsen. Letzteres ist auch sonst bei den Basitemporalia eine gewöhnliche Erscheinung. So verwächst bei den Reptilien das Parasphenoíd (und damit auch die Basitemporalia) schon sehr frühzeitig mit dem Basisphenoid, wie: es besonders GAUPP ${ }^{2}$ ) für Lacerta betont. Und dasselbe ist nach GAUPP auch mit den „Säugerpterygoiden" von Echidna der Fall.

GAUPP hat für die Pterygoidfrage Wert gelegt auf den Verlauf des Nervus vidianus s. parabasalis. Bei vielen Säugetieren wird der Canalis vidianus (parabasalis), welcher diesen Nerv enthält, von Schädelbasis und Pterygoid gebildet. Die Tatsache, dass bei Echidna anstatt des Pterygoid der von ihm gefundene neue Knochen an die Begrenzung des Kanales teilnimmt und bei Lacerta das Parasphenoid diese Stelle einnimmt, betrachtet GAuPP als eine Stütze für seine oben erwähnte Auffassung. über die Natur des Säugerpterygoid. Wie aber Fuchs mit Recht bemerkt, ist dieses Argument wenig. überzeugend, umsomehr weil GAUPP selbst gefunden hat, dass der Kanal bei Schildkröten vom Pterygoid gebildet wird, und er von diesem Knochen der Schildkröten sogar bemerkt, dass er grosse Aehnlichkeit mit dem Pterygoid von Echidna aufweist. Dass der Zustand bei Schildkröten demjenigen der Säugetiere nicht homolog ist, ist wahrscheinlich; er zeigt aber die Möglichkeit, dass bei Reduktion des Parasphenoid (bezw. der Basitemporalia) die Begrenzung des Kanales vom Pterygoid übernommen wird und es liegt kein Grund vor, diese Möglichkeit für die Säugetiere in Abrede zu stellen.

Der oben erwähnte Talpa-Embryo zeigt das folgende Verhältnis zwischen Nerv und Knochen (Fig. 1). Der Nerv läuft lateral vom Proc. tympanicus der Ventralfläche der Ala temporalis entlang um in der üblichen Weise über der Cranialfläche des Pterygoid seinen. Weg vorwärts zu verfólgen. Letzteres gibt auch FISCHER ${ }^{3}$ ) für ein. jüngeres Stadium von Talpa an und ich finde es auch bei Erinaceus. Der Nerv gelangt dann zwischen Ala und Lingula hindurch in das Cavum epiptericum, wo er in das Ganglion sphenopalatinum übergeht. Mit der Vorstellung, dass der Proc. tympanicus dem Basitemporale entspreche, ist dieser Zustand leicht in Uebereinstimmung zu bringen, indem man eine geringfügige Verschiebung dieses Knochens in medialer Richtung annimmt, wodurch'er aus dem Bereiche des Nerven gelangt ist. Auf ähnliche Unterschiede in den Beziehungen zwischen Nerven und Knochen hat auch GaupP selbst hingewiesen ${ }^{4}$ ).

Ich möchte nicht umhin noch auf eine Tatsache hinzuweisen, welche indirekt für die von mir verteidigte Deutung des Proc. tympanicus der Insectivoren zu sprechen scheint. Bei einigen Theromorphen hatte das Basisphenoid seitliche Fortsätze, welche denen der Insectivoren in Gestalt und Lage sehr ähnlich waren ${ }^{5}$ ). Bei Labidosaurus nun trugen diese Fortsätze nach Brollı beide eine Reihe kleiner Zähnchen. Die Deckkochennatur der Fortsätze erscheint dadurch zweifellos und es liegt nahe auch in ihnen Basitemporalia zu vermuten. Um so wahrscheinlicher wird dann dasselbe für die zwar zahnlosen, aber sonst so ähnlichen Proc. tympanici der Insectivoren.

Nur nebenbei will ich bemerken, dass auch die sogenannten Proc. tympanici der Alisphenoide der Marsupialia und einiger Insectivora vielleicht denselben Ursprung haben wie diejenigen des Basisphenoid. Ueber ihre Entwicklung ist wenig bekannt; WATSON, nach dessen Angabe sie bei Marsupialia wenigstens nicht knorplig angelegt werden, vergleicht sie mit den Echidna-Pterygoiden. Auf seine Ansichten komme ich weiter unten noch zurück.

Noch eine weitere Eigentümlichkeit des von mir untersuchten Schädels von Talpa deutet auf die Richtigkeit der Homologisierung von Säuger- und Reptilienpterygoid hin. Von der Ventralfläche des knöchernen Basisphenoid erhebt sich jederseits und zwar unmittelbar lateral vom vorderen Rande

1) Im Sinne eines Mischknochens zweiter Ordnung von Fuchs (Arch. f. Anat. und Entw.-Gesch., 1909, Suppl.-Bd., S. 50). Es wäre dies dann ein Beispiel der von GAUPP „Primordialfusion” genannten Erscheinung.

2) Anat. Anz., Bd. XXVII, 1905.

3) Anat. Hefte, 1. Abt., Bd. XVII, 1901.

4) Semon's Forschungsreisen, 1. c., S. 745.

5) Brolli, Palaeontographica, Bd. LI, 1904-05, S. 51 ff.; CASE, Bull. Amer. Mus. of Nat. Hist., vol. XXVIII, 1910, S. 163. 
des Proc. tympanicus ein kleiner, aber deutlicher knorpliger Höcker oder kurze Leiste, welche sich terminal dem verknorpelten Rande des Pterygoid anlegt (Fig. 1). Obgleich Gelenkspalten zwischen ihnen und den Pterygoiden fehlen, bin ich geneigt diese Fortsätze als rudimentäre Proc. basipterygoidei zu betrachten. Es besteht jedoch die Schwierigkeit, dass sie ganz median von den Nervi parabasales liegen, während diese Nerven bei den Lacertiliern unter den Proc. basipterygoidei entlang gehen. Eine Verschiebung der Nerven ist zwar möglich; Gründe für eine solche lassen sich aber nicht angeben und ich möchte mich daher noch nicht mit Bestimmtheit für die Homologie aussprechen.

Soweit mir bekannt, sind nur in einem anderen Falle bei Insectivoren Proc. basipterygoidei beschrieben worden und zwar von BROOM ${ }^{1}$ ) bei einer neugeborenen Chrysochloris hottentota in der folgenden Weise: "The basisphenoid is chiefly remarkable for the large membranous exostosis which forms a large process extending downwards and outwards. This process may be regarded as a basisphenoidal process comparable to the basisphenoidal process of many reptiles in having a true articulation with the pterygoid." Aus dieser Beschreibung wird aber nicht deutlich, ob BROOM dasselbe gesehen hat wie ich bei Talpa oder vielmehr die auch bei Chrysochloris vorhandenen Proc. tympanici,

Die Anwesenheit bei mehreren höheren Säugetieren eines Knochens, welcher als Basitemporale zu deuten ist, ist also ein Beweis, dass das Pterygoid der Mammalia mit Recht seinen Namen trägt. Mit diesen beiden Skelettstücken, Basitemporale und Pterygoid, sind die beiden oben erwähnten Knochen der Monotremen, von GAUPP als Parasphenoid $(A)$ und Reptilienpterygoid $(B)$ gedeutet, wohl ohne Zweifel identisch. Sowohl GAUPP wie WATSON halten das Säugerpterygoid für identisch mit dem erstgenannten Knochen $(A)$. Nach WATson ist dieser Knochen $A$ demnach auch dem Reptilienpterygoid homolog, während er $B$ in dem Epipterygoid der Reptilien und in dem Alisphenoid der höheren Säugetiere zurückzufinden glaubt: besonders der oben erwähnte Proc. tympanicus des Alisphenoid habe mit dem Echidnapterygoid Aehnlichkeit.

Die verschiedenen Ansichten lassen sich in der folgenden Weise tabellarisch darstellen:

\begin{tabular}{|c|c|c|c|}
\hline & REPTILIEN & MONOTREMEN & UEBRIGE SAUGETIERE \\
\hline $\begin{array}{l}\text { ältere Auffassung: } \\
\text { nach GAUPP: }\end{array}$ & $\begin{array}{l}\text { Pterygoid } \\
\text { Parasphenoid } \\
\text { (Reptilien)pterygoid }\end{array}$ & $\begin{array}{l}\text { Knochen } B \\
\text { Knochen } A \\
\text { Knochen } B\end{array}$ & $\begin{array}{l}\text { Pterygoid. } \\
\text { (Säuger)pterygoid }\end{array}$ \\
\hline nach WATSON: & $\begin{array}{l}\text { Pterygoid } \\
\text { Epipterygoid p.p. }\end{array}$ & $\begin{array}{l}\text { Knochen } A \\
\text { Knochen } B\end{array}$ & $\begin{array}{l}\text { Pterygoid } \\
\text { Alisphenoid p.p. }\end{array}$ \\
\hline nach mir: & $\begin{array}{l}\text { Basitemporale } \\
\text { Pterygoid }\end{array}$ & $\begin{array}{l}\text { Knochen } A \\
\text { Knochen } B\end{array}$ & $\begin{array}{l}\text { Proc. tympanicus des Basisphenoid, u.s. w. } \\
\text { Pterygoid }\end{array}$ \\
\hline
\end{tabular}

Ich weiche daher sowohl von GAUPP wie von WATSON ab, indem ich, in Uebereinstimmung mit der gewöhnlichen Ansicht den Knochen $B$ der Monotremen für das Säugerpterygoid halte, und zwar schon deshalb, weil ich ihn auf den auch von GAUPP hervorgehobenen Gründen als dem Pterygoid der Reptilien homolog ansehe. Dazu kommen aber die folgenden speziellen Argumente:

1. Sowohl bei Echidna wie bei Ornithorhynchus liegt $A$ dorsal vom Palatinum und von $B$, während letztgenannter Knochen sich in seinem vorderen Abschnitt mehr oder weniger unter das Palatinum schiebt (GAUPP, WATSON). Hierin stimmt, wie FuchS betont hat und ich bei Erinaceus und Talpa bestätigt finde, das Pterygoid der höheren Säuger mit dem Knochen $B$ überein.

2. Der Knochen $A$ verwächst bei den Monotremen frühzeitig mit dem Sphenoid und zeigt darin also Uebereinstimmung mit dem Proc. tympanicus der Insectivoren.

Aber auch für die entgegengesetzte Meinung GAUPP's sind gute Gründe anzuführen:

1. Der Knochen $A$ liegt weiter oral als $B$, ebenso wie das Pterygoid der Insectivoren sich oral vom Proc. tympanicus befindet. Hiermit steht es im Zusammenhang, dass $B$ bei Echidna an der Begrenzung der Paukenhöhle teilnimmt, wie das Basitemporale.

2. GAUPP hat nachdrücklich auf die ontogenetisch späte Anlage von $B$ hingewiesen und dasselbe ist, wie oben gezeigt worden, für den Proc. tympanicus der Insectivoren der Fall.

1) Proc. Zoolog. Soc. London, 1916, S. 456. 
Wenn auch die in der Tabelle erwähnte Ansicht mir die wahrscheinlichere vorkommt, so ist es daher doch bis jetzt nicht möglich, die Homologie der beiden Knochen der Monotremen mit Sicherheit zu bestimmen.

Der oben erwähnte Fund von rudimentären Proc. basipterygoidei ist, falls sie sich bestätigt, auch in "anderer Beziehung wichtig. Ich denke hierbei an die Alisphenoidfrage. Bekanntlich hat GAUPP auch hierin neue Ansichten geäussert, indem er die Ala temporalis des Säugerschädels auf den Proc. basipterygoideus der Reptilien zurückführt ${ }^{1}$ ). Wegen der bei mehreren Säugetieren nachgewiesenen gesonderten Entstehung der Ala temporalis oder der Lamina ascendens derselben weist es auch auf die Möglichkeit hin, dass sie ein Derivat des Palatoquadratknorpels sei, vielleicht die Columella cranii (Epipterygoid), wenn er das auch selbst als unwahrscheinlich betrachtet. Später haben dann BROOM ${ }^{2}$ ), FUCHS $^{3}$ ) und GREGORY ${ }^{4}$ ) diese letztgenannte Homologie (welche übrigens schon viel früher von RATHKE u. A. ausgesprochen wurde) ausführlicher begründet. Auf die ganze Frage möchte ich hier nicht eingehen, aber nur betonen, dass das Vorhandensein von besonderen Proc. basipterygoidei bei Talpa mit der erstgenannten Deutung von GAUPP nicht im Einklang steht und die Alisphenoid-EpipterygoidHomologie dadurch an Wahrscheinlichkeit gewinnt.

1) Anat. Hefte, Bd. XIX, H. 1, 1902, S. 159; Semon's Forschungsreisen, 1. c., S. 704.

2) Proc. Linn. Soc. N.-S.-Wales, vol. XXXIV, 1909, S. 105.

3) Anat. Anz., Bd. XXXVI, 1910, S. 33.

4) Journ. of Morphology, vol. XXIV, 1913, S. 1. 